\title{
Real-time high frame rate cardiac B-Mode and tissue Doppler imaging based on multiline transmission and multiline acquisition
}

\author{
Alessandro Ramalli, Alessandro Dallai, Francesco Guidi, Luca Bassi, \\ Enrico Boni, Ling Tong, Giuseppe Fradella, Jan D'hooge, and Piero Tortoli
}

\begin{abstract}
Cardiovascular diseases, the leading cause of death in the world, are often associated with the dysfunction of the left ventricle. Even if, in clinical practice, the myocardial function is often assessed through visual wall motion scoring on B-mode images, quantitative techniques have been introduced, e.g. ultrasound tissue Doppler imaging (TDI). However, this technique suffers from the limited frame rate of currently available imaging techniques that needs to be balanced with the field of view.

High frame rate (HFR) cardiac imaging has been recently tested off-line by simultaneously transmitting multiple focused beams into different directions and acquiring raw channel data into a PC. Several image lines were then reconstructed from the echoes of each transmission event. The same approach has been used to increase the TDI frame rate without restricting the field of view.

This paper demonstrates the real-time feasibility of multiline transmission and acquisition methods for both HFR cardiac BMode and tissue Doppler imaging. These approaches have been implemented on the ULA-OP 256 research scanner, by taking care that the related resources were optimally exploited for these new applications. The obtainable performance in terms of image quality and frame rate has also been investigated. Experiments performed with a 128-element phased array probe show, for the first time, that real-time B-mode imaging is feasible at up to 1150 $\mathrm{Hz}$ without significant reduction in image quality or field-of-view. The implementation of a real-time TDI algorithm allowed obtaining TDI images with a frame rate of $288 \mathrm{~Hz}$ for a $90^{\circ}$-wide field-of view. Finally, in-vivo examples demonstrate the feasibility and the suitability of the method in clinical studies.
\end{abstract}

Index Terms - Multiline transmission, tissue Doppler imaging, real-time, cardiac imaging, high frame rate imaging, multiline acquisition.

This work was supported by the Italian Ministry of Education, University and Research (PRIN 2010-2011) and from the European Union's Horizon 2020 research and innovation programme under the Marie Skłodowska-Curie grant agreement No 786027 (ACOUSTIC).

A. Ramalli is with the Laboratory of Cardiovascular Imaging and Dynamics, Department of Cardiovascular Sciences, KU Leuven, 3000 Leuven, Belgium but he was with the Department of Information Engineering, University of Florence, 50139 Florence, Italy when the system was implemented.

A. Dallai, F. Guidi, L. Bassi, E. Boni and P. Tortoli are with the Department of Information Engineering, University of Florence, 50139 Florence, Italy.

L. Tong is with SuperSonic Imagine, 13857 Aix-en-Provence, France.

G. Fradella is with the Cardiology Unit, Careggi University Hospital, 50010 Florence, Italy.

J. D'hooge is with the Laboratory of Cardiovascular Imaging and Dynamics, Department of Cardiovascular Sciences, KU Leuven, 3000 Leuven, Belgium.

\section{INTRODUCTION}

Cardiovascular diseases remain the leading cause of death in the world and thus have a tremendous impact on our society [1], [2]. Since major diseases (including congestive heart failure, coronary artery disease, hypertension, cardiomyopathies, ventricular dyssynchrony and others) are associated with (regional) dysfunction of the left ventricle [3], the accurate assessment of ventricular function during all phases of the heart cycle is fundamental. This goal is facilitated by ultrasound (US) imaging, which is in general appreciated for bedside applicability, good temporal resolution, real-time operation, low cost and absence of ionizing radiation.

Current standard clinical care is still largely based on US BMode imaging approaches, in which the region of interest is scanned line-by-line. The resulting frame rate is typically lower than desirable in cardiac applications $(\leq 100 \mathrm{~Hz})$ and may lead to loss of important diagnostic or prognostic information [4].

Even if, in clinical practice, the myocardial function is still often assessed through visual wall motion scoring, quantitative techniques have been introduced [5]-[8]. Myocardial strain and strain rate values [9]-[11] can be obtained by either color tissue Doppler imaging (color TDI) [5], [12] or speckle tracking echocardiography (STE) [13]. The latter technique, although currently used in high-end commercial scanners, is known to suffer from reduced sensitivity to motion. On the other hand, TDI is not affected by this problem, and has been demonstrated as an excellent tool for early detection of myocardial dysfunction, for prognosis and for follow-up evaluations of myocardial function after different medical and surgical treatments [8], [10], [14]-[16]. However, TDI exploits the same principles of color flow imaging and thus suffers for the same restrictions, i.e. low frame rate and/or limited field of view [17].

Recently, high frame rate (HFR) ultrasound imaging is getting increasing interest in echocardiography for its capability of capturing rapid cardiac events. In order to reduce the number of transmission events, high-end ultrasound scanners transmit beams with wide opening angle together with parallel receive beamforming (or multiline acquisition, MLA) [18], [19]. This approach reconstructs several lines for each transmission event and as such increases the frame rate, 
but it suffers from a degradation of lateral resolution and artifacts [20]. These drawbacks are typically mitigated, with an unavoidable frame rate reduction, by synthetic aperture or coherent spatial compounding methods [21]-[25]. In particular, it was shown that diverging wave compounding can produce high-contrast, high-resolution B-mode images [26][28], as well as simultaneous tissue Doppler images when motion compensation was integrated [29].

An alternative approach is the so-called multiline transmission (MLT), i.e. a high frame rate imaging method, particularly suitable for cardiac imaging, based on the simultaneous transmission of multiple focused beams into different directions; it enables an increase of the frame rate by a factor equal to the number of MLT beams [30]-[32].

Our research groups got considerable knowhow and experience in MLT imaging: simulations [23] and off-line experiments [33] demonstrated that the MLT approach increases the frame rate without significantly compromising the spatial resolution, the contrast and the signal-to-noise ratio. It has also been shown that either the field-of-view or the frame rate of TDI may benefit from MLT beamforming [17]. However, the application of these approaches in clinical routine is still hindered by the lack of real-time operation, as in previous studies B-Mode and TDI images were reconstructed off-line. Indeed, HFR imaging in real-time by MLT and MLA requires: in transmission (TX), the use of arbitrary waveform generators for excitation of the different transducer elements with non-identical electric pulses; in reception (RX), the implementation of parallel beamforming methods, which are highly demanding in terms of data rate, onboard memory, and computational load.

Therefore, with the goal of future intensive clinical trials, this work, whose early steps were presented in [34], demonstrates the real-time feasibility of MLT and MLA methods for HFR cardiac B-Mode and tissue Doppler imaging. Both above methods have been implemented on the ULA-OP 256 research scanner [35], [36], by taking care that the related resources were optimally exploited for these new applications. Implementation details and the achieved performance in terms of frame rate and image quality are here presented together with in-vivo examples of both B-mode and TDI acquisitions.

The paper is organized as follows: section II reviews the basics of MLT and MLA beamforming, the algorithm for TDI processing, a detailed description of the real-time implementation on the ULA-OP 256 scanner as well as the parameters used to estimate the processing speed and image quality; section III shows the experimental results that are finally discussed in section IV.

\section{METHODS}

\section{A. Multiline transmission and multiline acquisition}

Multiline acquisition, also referred to as parallel beamforming, was proposed in the literature to solve the limited frame rate achieved with standard ultrasound imaging [18], [19], [22]. It consists in the transmission of wide beams (e.g. plane waves or defocused waves) followed by the simultaneous reconstruction of $n M L A$ lines. Conversely, multiline transmission (MLT) consists in simultaneously transmitting $n M L T$ beams, which are spatially distributed over the volume of interest [30], [31].

In this paper, both MLT and MLA are simultaneously implemented to exploit the strengths of both methods, so that the frame rate becomes:

$$
F R=\frac{P R F}{n T X}=\frac{P R F \cdot n M L T \cdot n M L A}{n \text { Lines }}
$$

where $\mathrm{PRF}=1 / \mathrm{PRP}$ is the pulse repetition frequency, $n T X$ is the number of transmission events needed to scan the whole region of interest and nLines, hereinafter assumed equal to 128 , is the number of lines per image frame.

To simultaneously transmit multiple beams into different directions, non-identical electric pulses must be applied to the elements of the phased array transducer. The excitation waveform for a given element is given by the sum of the pulses, each properly delayed, that would be used on that element to generate conventional focused single line transmission (SLT) beams along the directions of the different MLT lines:

$$
\begin{aligned}
\theta_{T X}(i, j)=-\frac{\theta_{T}}{2} & +\frac{(n M L A-1)}{2} \cdot \theta_{S} \\
& +(i-1) \frac{\theta_{T}}{n M L T} \\
& +(j-1) \cdot n M L A \cdot \theta_{S}
\end{aligned}
$$

where $\theta_{T}$ is the sector angle, $\theta_{S}=\theta_{T} /$ nLines is the stepangle between lines, $i \in[1, n M L T]$ is the index of the simultaneous MLT beams and $j \in[1, n T X]$ is the index of the transmission event, see Fig. 1 for a schematic example. Similarly, the directions of the reconstructed lines are computed as follows:

$\theta_{R X}(i, j, k)=\theta_{T X}(i, j)+\left[(k-1)-\frac{(n M L A-1)}{2}\right] \theta_{S}$

where $k \in[1, n M L A]$ is the index of the MLA lines.

As an example, Fig. 2 shows the pulses that would be applied to the elements of a phased array to independently (Fig. 2a-d) or simultaneously (Fig. 2e) transmit ultrasound waves into four directions.

\section{B. Virtual source}

It is worth noting that the sum of pulses leads to constructive interferences (e.g. look at the signal on the central element in Fig. 2e) that may exceed the maximum available transmitter output or the maximum admissible voltage on the transducer element. In these cases, the signals of all active elements must be divided by a normalization coefficient $\alpha$ so that all the signals fit the maximum limits. The higher $\alpha$, the lower is the energy transmitted along each direction. In general, if no countermeasures are taken, the value of $\alpha$ is equal to $n M L T$ since full constructive interference happens in the central element of the active aperture, while out of phase 
interference happens for the other elements. In particular, this happens when, in the reference system $(x, z)$ corresponding to lateral and axial direction, respectively, the source of the imaging sector is at $(0,0)$. Full constructive interference can be limited by imposing phase and/or time delays between different MLT beams [37] or - as proposed in this paper - by setting the source of the imaging sector on the back of the probe surface at $(0, \partial z)$, see Fig. 1 bottom panel. In such a way, constructive interference is shared among several elements bringing the maximal amplitude of the signal down and hence requiring lower $\alpha$ values. In general, when any MLT approach is implemented $\alpha$ is always a number greater than 1 and it depends on $n M L T, \theta_{T}$, the transmitted signal length, the transmission apodization, and $\partial z$. The only way to avoid such normalization is implementing MLT in time division multiplexing but it lengthens the transmission length and hence increases the image blind region. The use of a virtual source, not only maximizes the energy transmitted along each direction, but also mitigates acoustic safety issues in the near field, where the mechanical index is higher due to the overlap of beams [37], [38].

\section{Tissue Doppler processing}

TDI measures the velocity of myocardium relative to the transducer in a similar way as conventional color flow imaging. For each image point, a packet of $N_{p}$ complex samples $s_{d, l, m \ldots m-N_{p}+1}$ is extracted from $N_{p}$ subsequent acquired frames, where $s$ is the quadrature (IQ) demodulator output, $d, l, m$ are the depth, line and frame indexes,
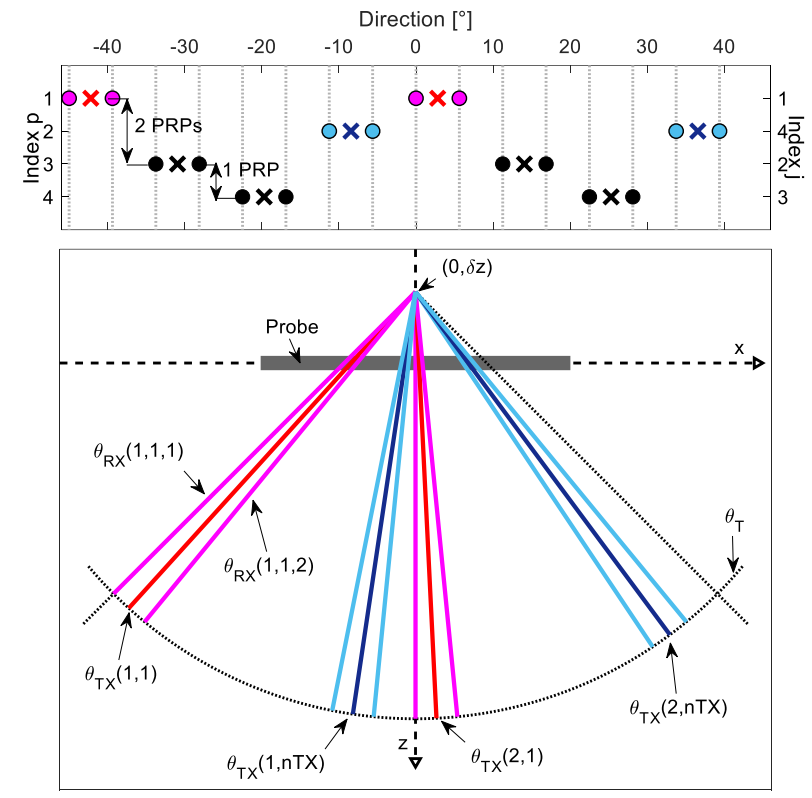

Fig. 1 Schematic example of the multiline transmission and multiline acquisition directions and of the transmission event ordering for nMLT $=2$, $\mathrm{nMLA}=2$ and $\mathrm{nTX}=4$. The top panel shows the direction of the MLT beams (crosses) and of the MLA lines (dots) for each transmission event (Index p), sorted according to (6). The maximum time lag between consecutive lines is 2 PRPs, while it would have been nTX following the order of the increasing steering directions (j). The bottom panel shows the sketch of the reference system $(\mathrm{x}, \mathrm{z})$, the probe, the virtual source position $(0, \partial z)$ and the directions of MLT beams and MLA lines for the first two transmission events. respectively.

The selected sequence still presents residual flow signal and white bandwidth noise contributions that are removed by a low-pass filter, hereinafter called noise filter. The Kasai estimator [39] performs the integral of the phase changes to produce the one-step autocorrelation function, as follows:

$$
\begin{gathered}
\hat{R}_{d, l}(1)=\frac{1}{N_{p}-1} \sum_{m=0}^{N_{p}-2} s_{d, l, m-i}^{*} \cdot s_{d, l,(m-i-1)} \\
\hat{v}_{d, l}=\frac{c \cdot F R}{4 \pi f_{0}} \arg \left(\hat{R}_{d, l}(1)\right)
\end{gathered}
$$

where $\mathrm{c}$ is the speed of sound and $f_{0}$ is the central frequency of the received signal.

The matrix of the estimated velocities is spatially and temporally filtered to attenuate possible non-physiological sharp variations among the lines and/or the frames, typically due to noise. This filter is hereinafter referred to as space-time filter.

\section{Transmission event ordering}

In MLT imaging mode, given that one or more lines (in MLA mode) are reconstructed from each of the $n M L T$ beams, the final image is divided into $n M L T$ subsectors. If the order of the transmission events is not properly selected, the time lag between consecutive lines could induce artifacts. In particular, if the order is selected as in typical phased scans, i.e. at increasing steering directions, the time lag between consecutive lines is equal to one PRP, except at the edges of consecutive subsectors, where the time lag is equal to $n T X$ PRPs. Hence, when imaging anatomical structures that move rapidly, significant discontinuities may appear at the edges of each subsector. In this paper, as proposed in [40], the index of the transmission event $j$ in (2) and (3) is ordered as follows:

$$
j= \begin{cases}\frac{p+1}{2}, & \text { if } p \text { is odd } \\ n T X-\left(\frac{p}{2}-1\right) & , \text { if } p \text { is even }\end{cases}
$$

where $p \in[1, n T X]$ is the current PRP index. In this way, the time lag between consecutive lines is always equal to 2 PRPs except for the edge lines where it is 1 PRP, see Fig. 1.

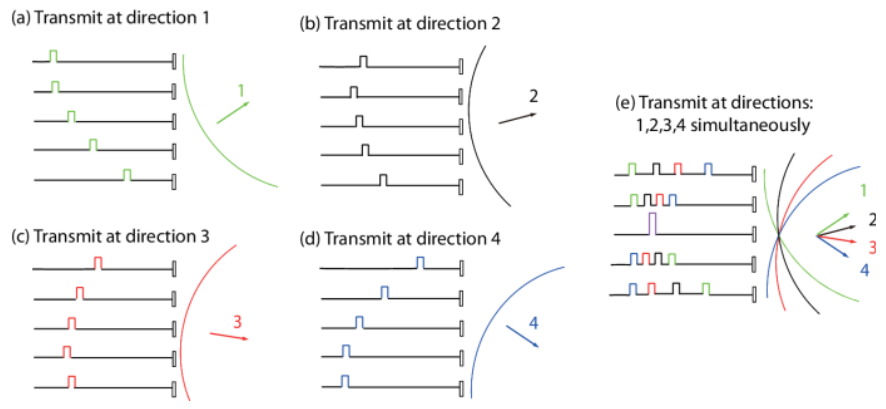

Fig. 2 Pulses to be applied on the elements of a phased array probe in order to generate four focused lines (a), (b), (c), (d) either consecutively, or simultaneously (e). 


\section{E. ULA-OP 256 real-time operation}

As illustrated in Fig. 3, ULA-OP 256 was equipped with a 128-element phased-array probe, 4 Front-End (FE) boards, each controlling 32 TX-RX channels, and one Master Control (MC) board. Each FE board contains one Field Programmable Gate Array (FPGA - ARRIA V GX, Altera, San Jose, CA, USA), and two multicore digital signal Processors (DSPs TMS320C6678, Texas Instruments, Austin, TX, USA). The MC board collects the data independently produced by the FE boards, completes the processing by a further DSP and sends B-Mode and TDI images to the host PC.

\section{1) In transmission}

The need of transmitting different waveforms with different amplitudes to the 128 active probe elements, is met in ULA-OP 256 by arbitrary waveform generators and high voltage linear amplifiers. The waveforms are individually synthesized, for each probe element, by the FE FPGAs that produce sigma-delta modulated bitstreams [41] in the Low Voltage Differential Signaling (LVDS) format at $468 \mathrm{Mb} / \mathrm{s}$. The bitstreams are low-pass filtered to extract the baseband spectrum contained in the sigma-delta modulation and finally sent to the high voltage linear amplifiers (MAX14807 Maxim Integrated, San Jose, CA, USA).

\section{2) In reception}

The backscattered echoes are band-pass filtered (with selectable cut-off frequencies), low noise amplified with variable gain for time gain compensation (TGC), and analogto-digital converted by Analog Front-End chips (AFE5807, Texas Instruments, Austin, TX, USA) at 78 MSPS with a resolution of $12 \mathrm{bit}$.

The digital samples are re-sampled by the on-board FPGA at 19.5 MSPS; this frequency is roughly 4 times the Nyquist limit and could be reduced, but such oversampling allows improving dynamic resolution, reducing noise and relaxing the requirements of the anti-aliasing filter. The samples are then stored inside a double buffer memory which feeds up to 4 delay and sum (DAS) units, where the first stage of the beamformer is performed at radiofrequency. The memory collects fresh samples at the lower sampling rate while the
DAS simultaneously runs at 234 MSPS with data acquired in the previous PRP. According to the beamforming coefficients, the DAS unit delays the samples of every channel with improved temporal offsets thanks to an embedded 1:16 quadratic interpolator, then multiplies the result by the apodization factor and sums the channels together. Within one PRP, the DAS units are reprogrammed multiple times with different beamforming schemes so to process $n M L T \cdot n M L A$ lines, delivering an average cumulative output higher than 500 MSPS.

The partially beamformed data are transferred to the two on-board DSPs by means of two SerialRapidIO (SRIO) links, with a total $\mathrm{B}_{\text {IOFE }}$ bandwidth of $3.5 \mathrm{~GB} / \mathrm{s}$. The samples are initially collected inside external DDR memory modules and segmented in large circular buffers. The DSPs employ their 16 cores to move the data from the modules to internal buffers through Direct Memory Access (DMA) channels, one line at a time, and to massively process them with a total throughput of 1.4 GSPS. The samples of each line are quadrature demodulated according to the frequency of the transmitted beams, low-pass filtered by a cascade of four moving average filters and down-sampled to produce a set of 512 samples per line.

The samples processed by the $4 \mathrm{FE}$ boards are channeled into an SRIO link, with a $\mathrm{B}_{\text {IOMC }}$ bandwidth of $1.85 \mathrm{~GB} / \mathrm{s}$, and streamed to the MC Board. Here, another multicore DSP performs the last beamforming stage by summing together the samples coming from different boards. The same DSP, by means of the MLT/MLA module, rearranges the lines to obtain an ordered sequence of columns likewise they are represented in the display, by considering the ordering sequence described in (2), (3) and (6). Each B-Mode image is formed by calculating the power of the signal, applying a fixed threshold to reject noise and logarithmically compressing the result. The final amplitudes are mapped to 256 gray-levels for each pixel. The same baseband data are simultaneously processed according to TDI, according to the algorithm described in section IIC. In particular, the noise filter is implemented as a 3rd order IIR filter: its cutoff frequency is set by default to $300 \mathrm{~Hz}$, which roughly corresponds to 12

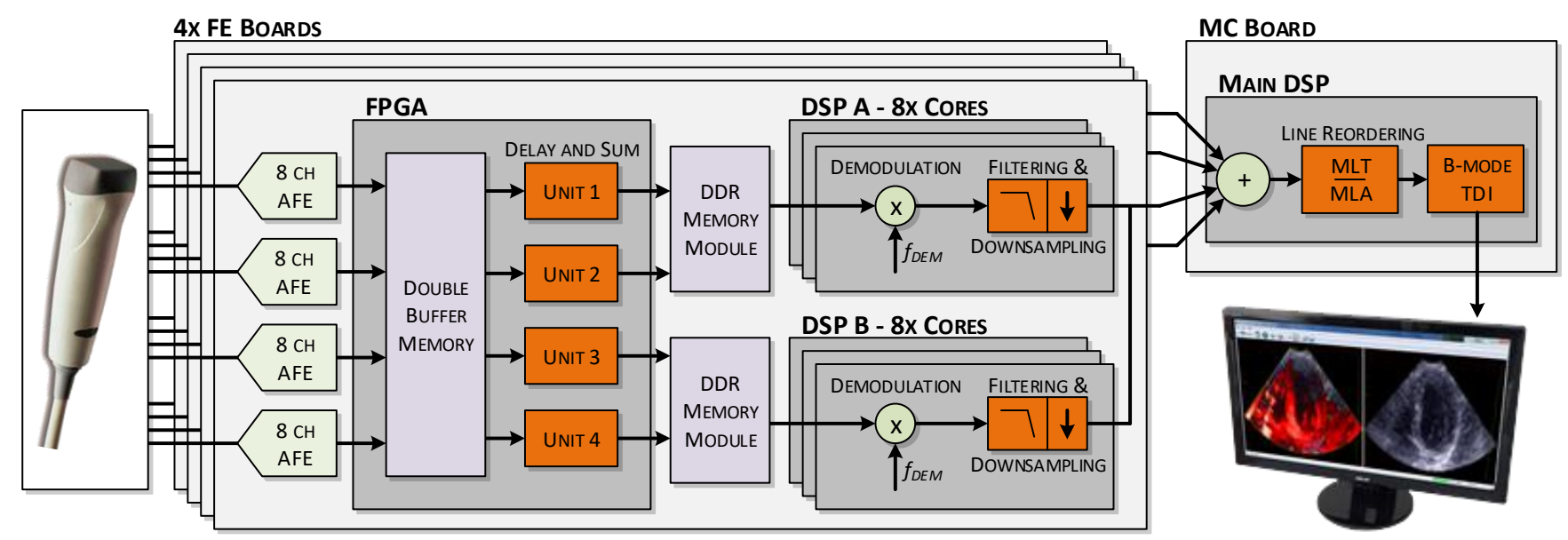

Fig. 3 Distribution across ULA-OP's boards of the main processing blocks involved during the reception phase. 
TABLE I

EXPERIMENTAL MODES

\begin{tabular}{|c|c|c|c|c|c|c|c|c|c|c|c|c|}
\hline Mode name & $\begin{array}{l}\text { MLT1- } \\
\text { MLA1 } \\
\end{array}$ & $\begin{array}{l}\text { MLT2- } \\
\text { MLA1 } \\
\end{array}$ & $\begin{array}{l}\text { MLT4- } \\
\text { MLA1 } \\
\end{array}$ & $\begin{array}{l}\text { MLT8- } \\
\text { MLA1 } \\
\end{array}$ & $\begin{array}{l}\text { MLT1- } \\
\text { MLA2 } \\
\end{array}$ & $\begin{array}{l}\text { MLT2- } \\
\text { MLA2 } \\
\end{array}$ & $\begin{array}{l}\text { MLT4- } \\
\text { MLA2 } \\
\end{array}$ & $\begin{array}{l}\text { MLT8- } \\
\text { MLA2 } \\
\end{array}$ & $\begin{array}{l}\text { MLT1- } \\
\text { MLA4 } \\
\end{array}$ & $\begin{array}{l}\text { MLT2- } \\
\text { MLA4 } \\
\end{array}$ & $\begin{array}{l}\text { MLT4- } \\
\text { MLA4 } \\
\end{array}$ & $\begin{array}{l}\text { MLT8- } \\
\text { MLA4 } \\
\end{array}$ \\
\hline nMLT & 1 & 2 & 4 & 8 & 1 & 2 & 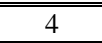 & 8 & 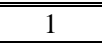 & 2 &  & 8 \\
\hline$\alpha$ & 1.0 & 1.7 & 1.9 & 2.0 & 1.0 & 1.7 & 1.9 & 2.0 & 1.0 & 1.7 & 1.9 & 2.0 \\
\hline nMLA & \multicolumn{4}{|c|}{1} & \multicolumn{4}{|c|}{$\frac{1}{2}$} & \multicolumn{4}{|c|}{4} \\
\hline nTX & 128 & 64 & 32 & 16 & 64 & 32 & 16 & 8 & 32 & 16 & 8 & 4 \\
\hline
\end{tabular}

$\mathrm{cm} / \mathrm{s}$ peak velocity, but it is programmable to be adjusted to different peak velocities. The space-time filter is performed through a $3 \times 3$ low-pass Gaussian filter followed by a $1^{\text {st }}$-order low-pass IIR having a programmable cutoff frequency between 10 and $100 \mathrm{~Hz} . N_{p}$ is made programmable and possible values are $4,8,16$. Since the raw estimated velocities could still be noisy, noise-suppression strategies can be enabled such as thresholding, persistence filtering, and $3 \times 3$ median filtering. The output velocity values are finally mapped to 256-level blue/red color-coded images which are overlaid on the B-mode frames. In addition, to more easily assess diseases such as ventricular dyssynchrony, the real-time software interface allows selecting up to 8 points of interest, whose temporal velocities profiles are displayed on distinct graphs.

All image frames processed by the MC board are transferred to a host $\mathrm{PC}$ and displayed on a monitor in real-time. Thanks to the USB 3.0 connection, a maximum throughput $\mathrm{B}_{\mathrm{USB}}$ of $300 \mathrm{MB} / \mathrm{s}$ can be achieved between ULAOP 256 and the host PC.

\section{F. Experiments}

\section{1) Experimental setup}

The ULA-OP 256 system was connected to a 128-element phased array probe PA230 (Esaote S.p.A., Florence, Italy), which has a $58 \%$ bandwidth centered at $2.1 \mathrm{MHz}$ and a 170 $\mu \mathrm{m}$ pitch.

The basic TX signal was a 1-cycle sine burst at $2 \mathrm{MHz}$, the peak amplitude of which was set to $64 \mathrm{~V}$. The focal depth was set to $6.5 \mathrm{~cm}$ and the apodization was a 96-element Hanning window to widen the transmitted beams and suppress sidelobes and thereby limit cross-talk between MLT beams. The above settings were used for each of the MLT beams and the excitation signal for each element was obtained as the sum of the properly delayed and weighted pulses, as described in section IIA. In RX a sinc dynamic apodization with $\mathrm{F} \#=2$ was implemented. ULA-OP 256 was programmed to transmit different sequences, including SLT, 2-, 4-, 8-MLT. In order to obtain a normalization factor $\alpha=2$ (arbitrarily chosen) in 8MLT, i.e. the worst case, $\partial z$ was found to be $-5 \mathrm{~mm}$. The same value was set for the other sequences and consequently, $\alpha$ resulted 1, 1.7, 1.9 for SLT, 2-, 4-MLT respectively. In reception, the ULA-OP 256 was programmed to reconstruct 1, 2-, 4-MLA lines for each transmitted beam, see TABLE I. Each of the 12 modes scanned a $90^{\circ}$ wide sector trough 128 lines and over a range of 1 to $15.4 \mathrm{~cm}$.

\section{2) Speed performance metrics}

In order to estimate the performance in terms of speed of the whole system, we evaluated: the maximum achievable pulse repetition frequency $\left(\mathrm{PRF}_{\mathrm{Max}}\right)$; the maximum frame rate during continuous real-time $\mathrm{B}$-mode imaging $\left(\mathrm{FR}_{\mathrm{Max}}\right)$; the average bandwidth at the beamformer output $\left(\mathrm{B}_{\mathrm{BF}}\right)$; the SRIO data transfer bandwidths on the $\mathrm{FE}$ ( $\left.\mathrm{B}_{\mathrm{IOFE}}\right)$ and on the $\mathrm{MC}$ ( $\left.\mathrm{B}_{\mathrm{IOMC}}\right)$ boards; and the data transfer bandwidth to $\mathrm{PC}$ on the USB link ( $\left.\mathrm{B}_{\mathrm{USB}}\right)$.

\section{3) Image quality metrics}

The experimental evaluation of the quality of the real-time reconstructed images was based on a commercial tissue mimicking phantom 040GSE (Cirs Inc, Norfolk, VI, USA), which includes wires as well as hyperechoic and anechoic cysts. For each mode, 300 frames were acquired and the related quadrature demodulated baseband data $(I Q)$ were postprocessed to extract quality performance metrics.

The tissue mimicking phantom B-Mode image shown in Fig. 4 allows identifying three $4 \times 4 \mathrm{~mm}$ regions:

- $\mathrm{S}_{\mathrm{ROI}}$ : the background region, selected at the focal depth;

- $\mathrm{C}_{\mathrm{ROI}}$ : the anechoic region, selected inside the anechoic cyst at the focal depth;

- $\mathrm{N}_{\mathrm{ROI}}$ : the noise region, i.e. an area selected outside the phantom borders, where the intensity of artifacts and background signals was very low; here, any contribution to the signal intensity can be ascribed to noise.

The signal-to-noise ratio (SNR), the contrast ratio (CR) and the contrast-to-noise ratio $(\mathrm{CNR})$ were then defined as follows:

$$
\begin{gathered}
\mathrm{SNR}=\frac{\int_{N_{R O I}} d S}{\int_{S_{R O I}} d S} \cdot \frac{\int_{S_{R O I}}|I Q(S)|^{2} d S}{\int_{N_{R O I}}|I Q(S)|^{2} d S} \\
\mathrm{CR}=\frac{\int_{C_{R O I}} d S}{\int_{S_{R O I}} d S} \cdot \frac{\int_{S_{R O I}}|I Q(S)|^{2} d S}{\int_{C_{R O I}}|I Q(S)|^{2} d S} \\
\mathrm{CNR}=\frac{2 \cdot\left(\mu_{S_{R O I}}-\mu_{C_{R O I}}\right)^{2}}{\sigma_{S_{R O I}}^{2}+\sigma_{C_{R O I}}^{2}}
\end{gathered}
$$

where $\mu_{S_{R O I}}$ and $\mu_{C_{R O I}}$ are the average amplitude values in $\mathrm{S}_{\mathrm{ROI}}$ and $\mathrm{C}_{\mathrm{ROI}}$, respectively, while $\sigma_{S_{R O I}}$ and $\sigma_{C_{R O I}}$ represent the standard deviations. It is worth highlighting that, in order to compute $\int_{N_{R O I}}|I Q(S)|^{2} d S$ in (7), IQ signals were first high pass filtered (in the direction of the frames) to remove the DCcomponent due to steady artifacts. Finally, the lateral resolution was estimated as the width at $-6 \mathrm{~dB}\left(\operatorname{Res}_{6 \mathrm{~dB}}\right)$ and $12 \mathrm{~dB}\left(\operatorname{Res}_{12 \mathrm{~dB}}\right)$ of the point spread function of the wire placed at $80 \mathrm{~mm}$, i.e. the one on the border of the anechoic cyst used 


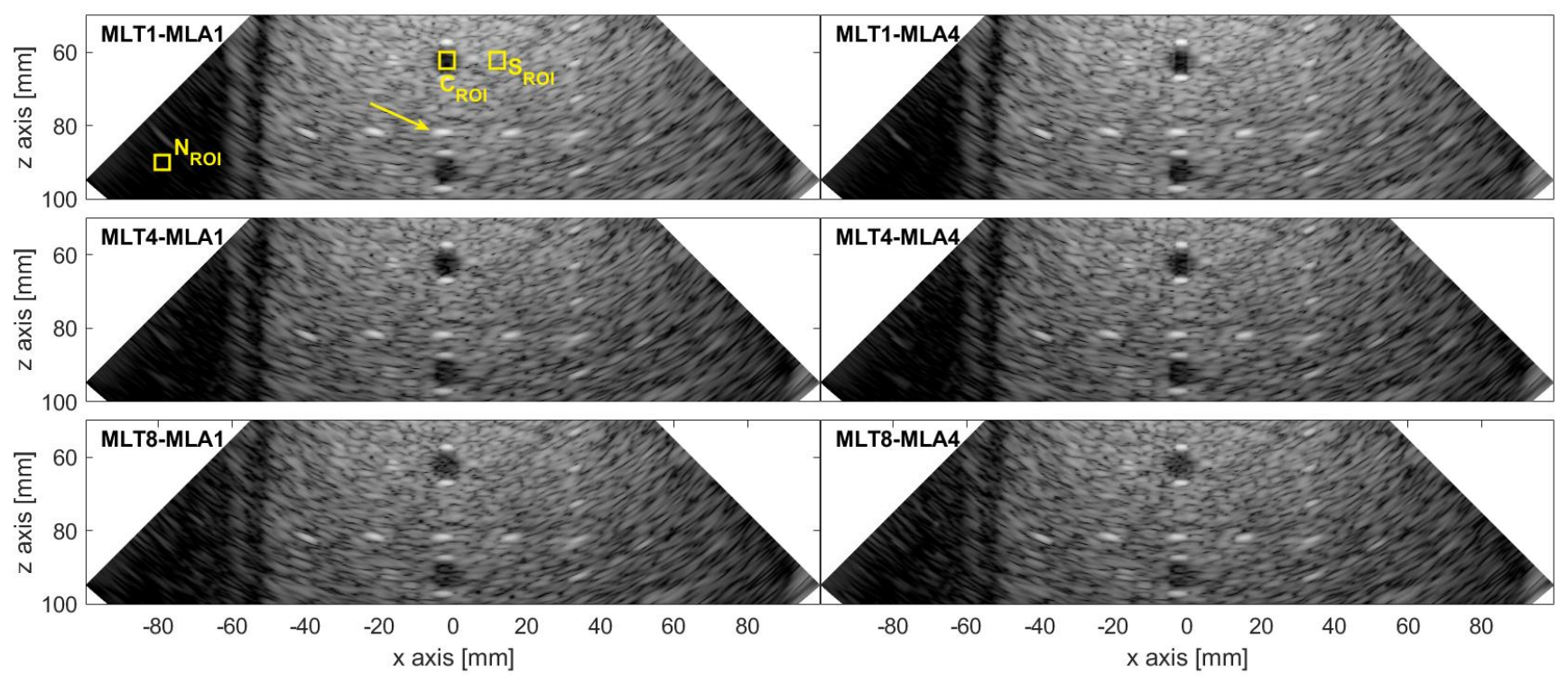

Fig. 4 Examples of B-mode images of the commercial tissue mimicking phantom obtained with different MLT/MLA modes. All the images are shown with the same threshold level and the dynamic range is $60 \mathrm{~dB}$. In the top left panel, $\mathrm{S}_{\mathrm{ROI}}, \mathrm{N}_{\mathrm{ROI}}$, and $\mathrm{C}_{\mathrm{ROI}}$ indicate the regions of interest used to estimate the $\mathrm{SNR}$, CNR, and CR. The arrow highlights the point spread function of the wire considered for the estimation of the lateral resolution.

for contrast estimation

\section{4) In-vivo experiments}

For in-vivo experiments the real-time interface of the ULAOP 256 (see Fig. 5) was split in three displays: the one at the top-right showed the B-mode image; the one at the top-left showed the TDI image overlaid to B-mode; the bottom display showed the time-traces of the velocities estimated at selected points of interest. Given that the peak velocities of the myocardium are expected to be higher than $10 \mathrm{~cm} / \mathrm{s}$ [15]-[17], the Nyquist theorem imposes a frame rate of minimally 520 Hz. Accordingly, the system was tested for TDI only in those modes, i.e. MLT4-MLA4, MLT8-MLA2, MLT8-MLA4, which enabled to meet this criterion. For these 3 selected modes the measured mechanical index (MI) was $0.76,0.73$ and 0.73 , while the thermal index (TI) at $\mathrm{PRF}_{\text {Max }}$ was 1.18, 2.14 and 2.01 respectively. According to [42], [43], these values were low enough for a safe (non-obstetric and nonneonatal) exam lasting up to 15 minutes.

The left ventricle of two healthy volunteers (males, 35- and 53-year old) were imaged by an expert cardiologist in twoand four-chamber views from an apical transducer position and clips of the ULA-OP 256 real-time interface were captured.

\section{RESULTS}

\section{A. Speed performance}

TABLE II shows the measured speed performance of the system for the different MLT-MLA modes. It is worth highlighting that for an unambiguous range of $15.4 \mathrm{~cm}$ (our maximum depth of interest) the maximum PRF is $5000 \mathrm{~Hz}$. All the modes achieved this limit except for MLT8-MLA4, which was limited by the maximum sample rate of the beamformer, which in this case was 528 MSPS.

As expected, all transfer bandwidths considered depended on the number of reconstructed lines per transmission event but remained, in general, well below the maximum sustainable transfer rates. In particular, in the worst case, $\mathrm{B}_{\mathrm{IOFE}}$ reached $2.11 \mathrm{~GB} / \mathrm{s}$ out of the $3.5 \mathrm{~GB} / \mathrm{s}$ sustainable by the two-channel SRIO transfer channel; B $\mathrm{IOMC}$ was lower than $1 \mathrm{~GB} / \mathrm{s}$ except for MLT8-MLA4 that approached the maximum limit of the one-channel SRIO, i.e. $1.85 \mathrm{~GB} / \mathrm{s}$; while the maximum required $\mathrm{B}_{\mathrm{USB}}$ was only $75 \mathrm{MB} / \mathrm{s}$, while its transfer channel could sustain up to $300 \mathrm{MB} / \mathrm{s}$.

Obviously, as the number of reconstructed lines per transmission event increases, the frame rate also increases. TABLE II shows that the minimum frame rate $(39 \mathrm{~Hz})$ is obtained with the standard scan mode (MLT1-MLA1), while the highest one (i.e. $1150 \mathrm{~Hz}$ ) is obtained with mode MLT8MLA4.

\section{B. Quality performance}

TABLE III shows the performance metrics for the tested modes. Here, a clear reduction in performance is evident when the number of simultaneously transmitted beams (nMLT) increases. Logically, the best performing modes were those based on MLT1: they achieved a SNR > 36dB, CNR>5.03, and

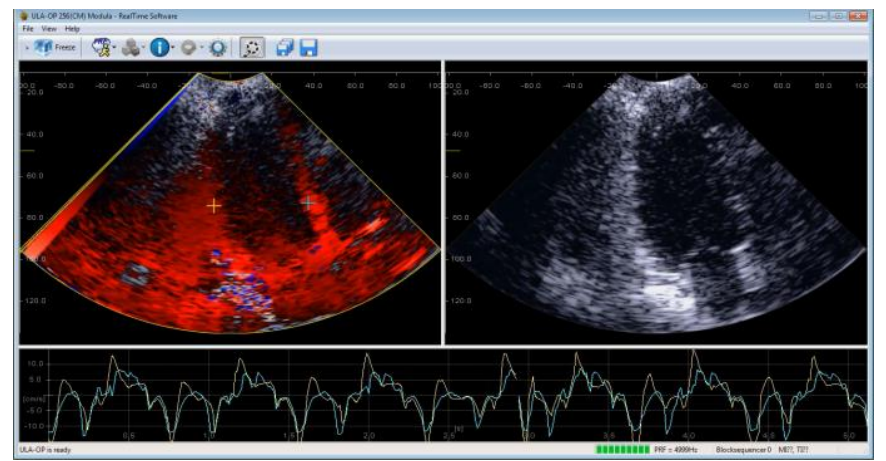

Fig. 5 Screenshot of the ULA-OP 256 real-time interface during a real-time TDI exam. On the right, the B-mode image; on the left, the TDI overlaid on B-mode image; on the bottom the time-traces of the velocities estimated at the selected points of interest. 
TABLE II

SPEED PERFORMANCE

\begin{tabular}{|c|c|c|c|c|c|c|c|c|c|c|c|c|}
\hline Mode name & $\begin{array}{l}\text { MLT1- } \\
\text { MLA1 } \\
\end{array}$ & $\begin{array}{l}\text { MLT2- } \\
\text { MLA1 } \\
\end{array}$ & $\begin{array}{l}\text { MLT4- } \\
\text { MLA1 } \\
\end{array}$ & $\begin{array}{l}\text { MLT8- } \\
\text { MLA1 } \\
\end{array}$ & $\begin{array}{l}\text { MLT1- } \\
\text { MLA2 } \\
\end{array}$ & $\begin{array}{l}\text { MLT2- } \\
\text { MLA2 } \\
\end{array}$ & $\begin{array}{l}\text { MLT4- } \\
\text { MLA2 } \\
\end{array}$ & $\begin{array}{l}\text { MLT8- } \\
\text { MLA2 } \\
\end{array}$ & $\begin{array}{l}\text { MLT1- } \\
\text { MLA4 } \\
\end{array}$ & $\begin{array}{l}\text { MLT2- } \\
\text { MLA4 } \\
\end{array}$ & $\begin{array}{l}\text { MLT4- } \\
\text { MLA4 } \\
\end{array}$ & $\begin{array}{l}\text { MLT8- } \\
\text { MLA4 }\end{array}$ \\
\hline $\mathbf{B}_{\mathrm{BF}}[\mathrm{MSPS}]$ & 18 & 36 & 72 & 143 & 36 & 72 & 143 & 287 & 72 & 143 & 287 & 528 \\
\hline B $_{\text {IOMC }}[\mathrm{GB} / \mathrm{s}]$ & 0.06 & 0.12 & 0.25 & 0.49 & 0.12 & 0.25 & 0.49 & 0.98 & 0.25 & 0.49 & 0.98 & 1.81 \\
\hline $\mathbf{B}_{\text {USB }}[\mathrm{MB} / \mathrm{s}]$ & 3 & 5 & 10 & 20 & 5 & 10 & 20 & 41 & 10 & 20 & 41 & 75 \\
\hline $\mathbf{F R}_{\text {Max }}[\mathrm{Hz}]$ & 39 & 78 & 156 & 313 & 78 & 156 & 313 & 625 & 156 & 313 & 625 & 1150 \\
\hline
\end{tabular}

The bottlenecks are highligthed in yellow.

TABLE III

QUALITY PERFORMANCE

\begin{tabular}{|c|c|c|c|c|c|c|c|c|c|c|c|c|}
\hline Mode name & $\begin{array}{l}\text { MLT1- } \\
\text { MLA1 } \\
\end{array}$ & $\begin{array}{l}\text { MLT2- } \\
\text { MLA1 } \\
\end{array}$ & $\begin{array}{l}\text { MLT4- } \\
\text { MLA1 } \\
\end{array}$ & $\begin{array}{l}\text { MLT8- } \\
\text { MLA1 } \\
\end{array}$ & $\begin{array}{l}\text { MLT1- } \\
\text { MLA2 } \\
\end{array}$ & $\begin{array}{l}\text { MLT2- } \\
\text { MLA2 } \\
\end{array}$ & $\begin{array}{l}\text { MLT4- } \\
\text { MLA2 } \\
\end{array}$ & $\begin{array}{l}\text { MLT8- } \\
\text { MLA2 } \\
\end{array}$ & $\begin{array}{l}\text { MLT1- } \\
\text { MLA4 } \\
\end{array}$ & $\begin{array}{l}\text { MLT2- } \\
\text { MLA4 } \\
\end{array}$ & $\begin{array}{l}\text { MLT4- } \\
\text { MLA4 } \\
\end{array}$ & $\begin{array}{l}\text { MLT8- } \\
\text { MLA4 } \\
\end{array}$ \\
\hline CNR & 5.08 & 4.97 & 4.58 & 3.86 & 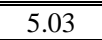 & 4.93 & 4.48 & 3.87 & "5.08 & 4.97 & 4.56 & 3.71 \\
\hline CR [dB] & 23.1 & 21.4 & 17.9 & 14.5 & 23.5 & 21.7 & 18.0 & 14.5 & 24.4 & 21.9 & 17.8 & 13.9 \\
\hline SNR [dB] & 36.5 & 33.8 & 28.0 & 27.2 & 36.4 & 33.7 & 28.0 & 27.0 & 36.2 & 33.4 & 27.7 & 26.8 \\
\hline $\operatorname{Res}_{6 \mathrm{~dB}}[\mathrm{~mm}]$ & 3.50 & 3.49 & 3.50 & 3.50 & 3.50 & 3.44 & 3.47 & 3.54 & 3.54 & 3.53 & 3.54 & 3.55 \\
\hline $\operatorname{Res}_{12 \mathrm{~dB}}[\mathrm{~mm}]$ & 4.98 & 4.94 & 4.97 & 5.00 & 4.95 & 4.89 & 4.91 & 4.99 & 4.76 & 4.73 & 4.73 & 4.72 \\
\hline
\end{tabular}

$\mathrm{CR}>23 \mathrm{~dB}$. On the other hand, the modes based on MLT8 performed the worst: $\mathrm{SNR}<27.2, \mathrm{CNR}<3.87$, and $\mathrm{CR}<14.5$. The lateral resolution was similar for all the modes; indeed, Res $_{6 \mathrm{~dB}}$ resulted $3.51 \pm 0.03 \mathrm{~mm}$, while $\operatorname{Res}_{12 \mathrm{~dB}} 4.88 \pm 0.11 \mathrm{~mm}$. This result is consistent with expectations since the lateral resolution in MLT imaging depends on the transmission/reception apodization scheme but not on the number of simultaneously transmitted beams [33], [44]-[46].

Qualitatively, the above numbers are confirmed by the images shown in Fig. 4: from left to right, i.e. for increasing $n M L T$, the overall brightness of the image reduces, while the artifacts level inside the anechoic cysts increases; from top to bottom, i.e. for increasing $n M L A$, only an increased pixelization of the image can be observer, especially close to the anechoic cyst borders and on the wires.

\section{Real-time TDI in-vivo images}

Fig. 6 shows the montage of four screenshots of the realtime interface of the ULA-OP 256 system that was captured during an exam of the left ventricle of the 53-year old healthy volunteer. The four panels, extracted from the accompanying clip 래, were captured during slow filling (A), during atrial contraction (B), during ventricular ejection (C), and at early diastole (D) of the same cardiac cycle. The B-mode, on the right of each panel, displays the four-chamber view obtained by the probe in the apical position. On the left of each panel, the Tissue-Doppler image, obtained with a packet size of 8 , is overlaid to the B-mode. In this example, the ULA-OP 256 was set in MLT4-MLA4 mode and the PRF was $5000 \mathrm{~Hz}$, hence the frame rate of the B-mode was $625 \mathrm{~Hz}$, while that of TDI was $78.1 \mathrm{~Hz}$. While the movement of the septum and the free wall of the left ventricle are properly overlaid to the B-mode, artifacts are visible deeper down in the image, especially in panel B, C and D. On the bottom panel of Fig. 6, the timetrend of the myocardial velocity is shown. The cyan-line refers to a point on the free-wall, while the yellow one to a point on the septum, as highlighted with crosses on the TDI images. A second accompanying clip shows the real-time interface of the ULA-OP 256 system that was captured during a twochamber view exam of the 35 -year old healthy volunteer.

\section{DISCUSSION AND CONCLUSION}

In this paper, we have reported the first real-time implementation of high frame rate cardiac imaging based on MLT combined with MLA. Now, the user can benefit from a direct feedback on the actual captured data; while, before, MLT imaging could only be achieved by SLT scouting, MLT blind recordings and offline post-processing.

In transmission, the real-time implementation exploits: the programmability of the arbitrary waveform generators of the ULA-OP 256 scanner to transmit MLT beams; the virtual source to maximize the energy transmitted along each beam; and the smart transmission event ordering to avoid motion artifacts at the edges of the subsectors of the images. In reception, the implementation of MLA needs high computational power that is provided by the two-stage beamformer, implemented on FPGA, enabling the beamforming of several lines within the same PRP and by the fast demodulator implemented on multicore DSPs. MLT imaging combined with MLA is preferable to other HFR methods providing comparable image quality and similar frame rates (in the order of hundreds of $\mathrm{Hz}$ ), due to its lower requirement in terms of beamforming speed. Indeed, diverging waves or synthetic aperture imaging require more computations, as all the pixels are beamformed multiple times for compounding; moreover, a full image - of at least 128 lines - must be produced every PRP. As an example, for MLT8MLA4 (the worst case) the required beamforming output rate is reduced by a factor 4 , but it is reduced even more for the other MLT/MLA modes. However, a different system architecture and a different processing pipeline, avoiding full RF DAS beamforming, would reduce the needed output data rate, e.g. by direct sampling IQ beamforming as implemented in Verasonics systems (Kirkland, WA, USA). This solution, although at the expense of a slight deterioration of image quality [47] and system flexibility, would also allow the real- 
time implementation of other HFR imaging methods such as diverging wave imaging.

The system was programmed in 12 different combinations of MLT and MLA (see TABLE I) and its effectiveness was assessed in terms of its performance in speed $\left(\mathrm{PRF}_{\mathrm{Max}}\right.$ and $\left.\mathrm{FR}_{\mathrm{Max}}\right)$ and image quality (CNR, CR, and SNR). Phantom experiments demonstrated that good B-mode images can be obtained at high frame rate in real-time. As expected, image quality decreased with increasing numbers of simultaneously transmitted beams (see TABLE III), while the multiline acquisition did not interfere with the processing pipeline apart from an increased pixelization of the image that could be evaluated only qualitatively. The performance drop in terms of SNR is partially explained by the different $\alpha$ values used in this experimental setup (see TABLE I) that limit the energy transmitted. The reduction of CNR and CR is due to artifacts generated by the cross-talk between beams in transmission and reception that becomes more severe with the number of transmitted beams as shown in [33], [44].

In most of the cases, in terms of speed performance, the frame rate was limited by the maximum depth, i.e. $15.4 \mathrm{~cm}$ in this work, that imposed a maximum PRF of $5000 \mathrm{~Hz}$. In those cases, the number of reconstructed lines could be increased, thus enhancing the image resolution in terms of pixels per inch, without impacting the frame rate. As an example, an MLT4-MLA7 could be implemented to achieve $625 \mathrm{~Hz}$ as in MLT4-MLA4 but providing a 224-line frame instead of a 128line one. The only exception was noted for mode MLT8MLA4, for which the PRF was limited to $4600 \mathrm{~Hz}$ since it saturated the output bandwidth of the beamformer. However, even this imaging mode still enabled continuous real-time imaging at a frame rate of $1150 \mathrm{~Hz}$ that, at the best of our knowledge, has never been achieved before. Although, the possible potential clinical benefits of such high frame rate are still debated [4], real-time MLT/MLA makes beamformed data immediately available; it could be useful both for further offline processing, speeding up the process from data capturing to final measurements (e.g. for speckle tracking [48]) and for direct online processing, as proposed in this paper for TDI. Indeed, the high frame rates achieved with MLT8-MLA4 (i.e. $1150 \mathrm{~Hz}$ ) and with both MLT4-MLA4 and MLT8-MLA2 (i.e. $625 \mathrm{~Hz}$ ) enabled the assessment of myocardial velocities up to 22 and $12 \mathrm{~cm} / \mathrm{s}$ respectively. The $12 \mathrm{~cm} / \mathrm{s}$ limit is typically sufficient; however, in some cases, myocardial peak velocities may be higher, hence aliasing may appear. This is evident also in the example shown in Fig. 6 D, where the mitral annulus moves too fast during early diastole. Possible alternative solutions to achieve even higher effective Nyquist velocities could be: the real-time implementation of pulse-package based MLT, at the expense of the output frame rate; the exploitation of a method based on diverging waves compounding [29], although not implemented in real-time. It is worth highlighting that such velocities can here be estimated on a wide image sector $\left(90^{\circ}\right.$ and 128 lines), in continuous realtime (i.e. without any acquisition interruption nor loss of data), and without pulse packeting. In this paper we also presented the real-time implementation of a tissue Doppler imaging method, that worked with an output frame rate of up to 288 $\mathrm{Hz}$, when using mode MLT8-MLA4 and setting $N_{p}=4$. Even if an experimental validation of tissue Doppler imaging with MLT has not been presented here, the method was thoroughly studied and validated in [17].

Furthermore, sample clips captured during in-vivo acquisitions on healthy volunteers demonstrated the feasibility of real-time TDI. In addition, the time traces of the myocardial velocity acquired in real-time at two points of interest, selected on the septum and on the free-wall of the left ventricle, were presented as well. In both the examples, the time-traces look physiologic, indeed they present typical trends of conventional pulse Doppler TDI traces, having clear peak systolic velocity $\left(S^{\prime}\right)$, as well as the early $\left(E^{\prime}\right)$ and late (A') peak diastolic velocities. Their values are comparable with those determined with a Vivid 7 scanner (GE Health care, Chicago, IL, USA) for an in-vivo independent study on 1266 healthy individuals, i.e. $\mathrm{S}^{\prime}=8.4 \pm 1.4, \quad \mathrm{E}^{\prime}=11.3 \pm 3.2 \quad$ and $\mathrm{A}^{\prime}=10.0 \pm 1.9$ (Table 4 in [49]). Moreover, the time-traces highlight that the two walls are moving in sync, as expected for a healthy individual.

The main limitation of MLT/MLA techniques applied to TDI applications is the presence of artifacts that, even if they do not jeopardize the B-mode image, may have a significant impact on the tissue Doppler image. Here, movements are detected even where they should not be detected, see Fig. 6 B and $\mathrm{C}$; this is due to the cross-talk between different beams, in MLT and MLA imaging, that generates artifact replicas of the strong echoes coming from the myocardium close to the mitral valve. In order to limit the level of artifacts, at least three solutions could be worth investigating: the filtered delay multiply and sum beamforming [50], a completely different transmission scheme, i.e. the transmission of diverging waves [51] and second harmonic imaging [28], [52]. The former was already demonstrated capable to reduce MLT/MLA artifacts [50] by exploiting the coherence of received echoes in the beamforming; however, it involves additional computational load that might impact on the maximum achievable frame rates in real-time. The transmission of several diverging waves with compounding in reception is gaining increasing interest for HFR imaging and was shown effective for several cardiac applications [26], [51], [27], [53], [29], [54]; even if it generates strong artifacts, due to the transmission of defocused beams, it is hard to predict their (either positive or negative) impact on TDI artifacts. Finally, second harmonic imaging inherently reduces artifacts due to grating lobes and was also shown, in a simulation study, to be effective on MLT imaging artifacts [52].

An additional limitation of TDI in the presented real-time implementation lies in the measurement of a single component of the displacement and in its dependency on the steering angle. This limitation could be solved, at the price of an increased computational load, by the implementation of twocomponent estimation techniques [55]-[57] and might improve the detection and localization of high strain regions. 


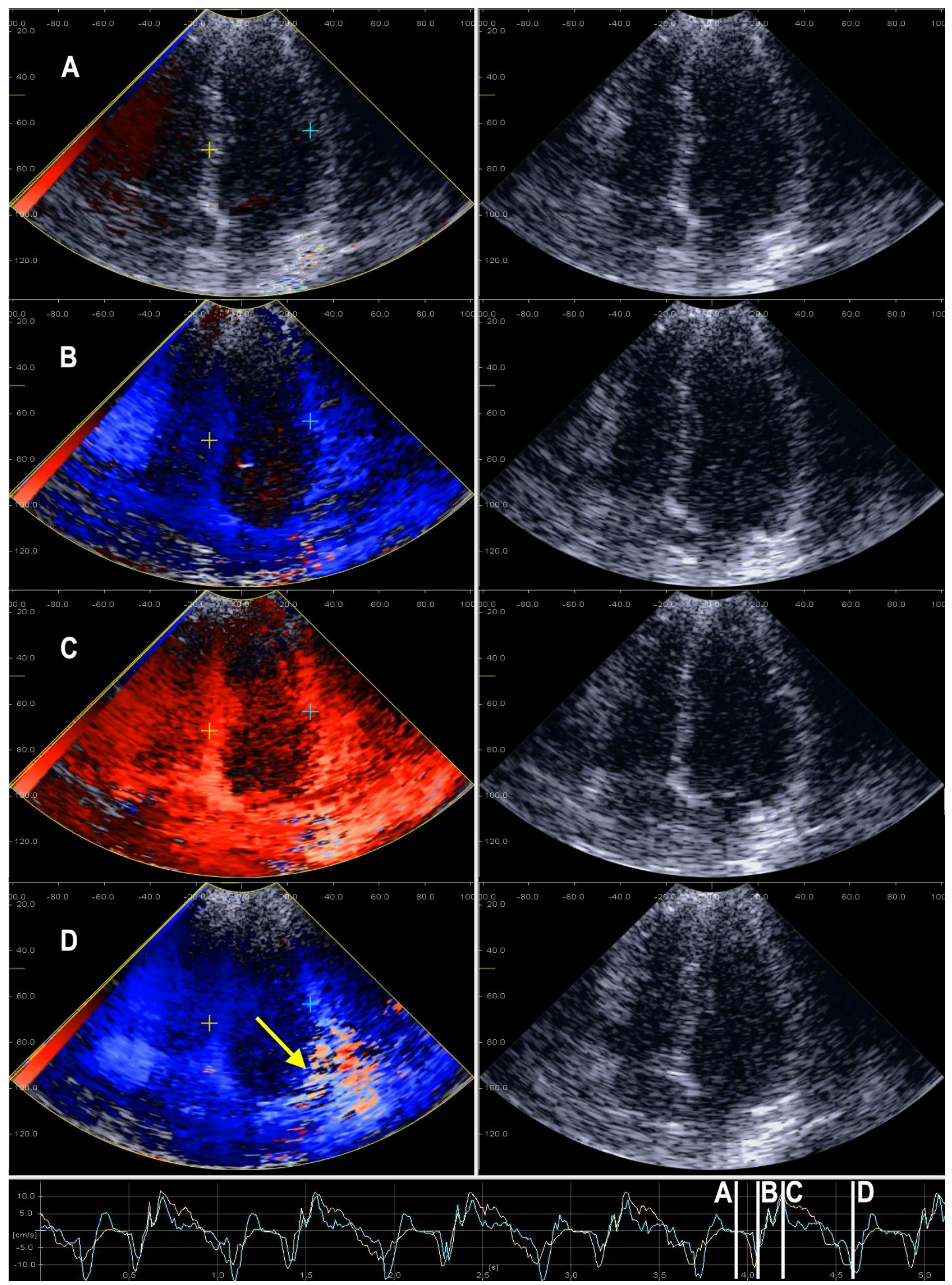

Fig. 6 Montage (extracted from the accompayning clip 트) of three screenshots of the ULA-OP 256 real-time interface during the exam of the left ventricle of the healthy volunteer. Each panel shows on the right the B-mode image, at $625 \mathrm{~Hz}$, obtained with 4MLT-4MLA, while on the left shows the TDI at 78.1 Hz overlaid on the B-mode image. The three panels were captured during slow filling (A), during atrial contraction (B), during ventricular ejection (C), and during early diastole (D) of the same cardiac cycle. The yellow line in (D) highlight the alising effect due to high myocardial peak velocities. The bottom panel shows the velocities on the septal wall (yellow) and on the left ventricular free wall (cyan) during the whole exam. 
In conclusion, we presented an innovative ultrasound system for imaging at very high temporal resolution with reasonable compromise in image quality but without compromising the field of view, thereby enhancing the diagnostic potential of cardiac ultrasound. The real-time implementation makes this tool ideal for clinical translation which is the topic of ongoing work. For example, the high frame rate, wide angle TDI data may enable a better detection of the heterogeneity of the onset of regional myocardial deformation (i.e. left ventricular dyssynchrony) which may help to unravel conduction disorders.

\section{REFERENCES}

[1] D. Mozaffarian et al., "Heart Disease and Stroke Statistics-2016 Update," Circulation, p. CIR.0000000000000350, Jan. 2015.

[2] N. Townsend, L. Wilson, P. Bhatnagar, K. Wickramasinghe, M. Rayner, and M. Nichols, "Cardiovascular disease in Europe: epidemiological update 2016," Eur. Heart J., vol. 37, no. 42, pp. 3232-3245, Nov. 2016.

[3] M. M. Redfield, S. J. Jacobsen, J. John C. Burnett, D. W. Mahoney, K. R. Bailey, and R. J. Rodeheffer, "Burden of Systolic and Diastolic Ventricular Dysfunction in the Community: Appreciating the Scope of the Heart Failure Epidemic," JAMA, vol. 289, no. 2, pp. 194-202, Jan. 2003.

[4] M. Cikes, L. Tong, G. R. Sutherland, and J. D'hooge, "Ultrafast Cardiac Ultrasound Imaging: Technical Principles, Applications, and Clinical Benefits," JACC Cardiovasc. Imaging, vol. 7, no. 8, pp. 812-823, Aug. 2014.

[5] G. R. Sutherland et al., "Color Doppler myocardial imaging: a new technique for the assessment of myocardial function," J. Am. Soc. Echocardiogr. Off. Publ. Am. Soc. Echocardiogr., vol. 7, no. 5, pp. 441-458, Oct. 1994.

[6] L. Hatle and G. R. Sutherland, "Regional myocardial function: a new approach," Eur. Heart J., vol. 21, no. 16, pp. 1337-1357, Aug. 2000.

[7] G. R. Sutherland, G. Di Salvo, P. Claus, J. D'hooge, and B. Bijnens, "Strain and strain rate imaging: a new clinical approach to quantifying regional myocardial function," J. Am. Soc. Echocardiogr., vol. 17, no. 7, pp. 788-802, Jul. 2004.

[8] C.-M. Yu, J. E. Sanderson, T. H. Marwick, and J. K. Oh, "Tissue Doppler Imaging: A New Prognosticator for Cardiovascular Diseases," J. Am. Coll. Cardiol., vol. 49, no. 19, pp. 1903-1914, May 2007.

[9] M. Dandel, H. Lehmkuhl, C. Knosalla, N. Suramelashvili, and R. Hetzer, "Strain and Strain Rate Imaging by Echocardiography - Basic Concepts and Clinical Applicability," Curr. Cardiol. Rev., vol. 5, no. 2, pp. 133-148, May 2009.

[10] M. Dandel and R. Hetzer, "Echocardiographic strain and strain rate imaging - Clinical applications," Int. J. Cardiol., vol. 132, no. 1, pp. 11-24, Feb. 2009.

[11] R. Jasaityte and J. D'hooge, "Strain rate imaging: fundamental principles and progress so far," Imaging Med., vol. 2, no. 5, pp. 547-563, Oct. 2010.

[12] J. D'hooge et al., "Regional Strain and Strain Rate Measurements by Cardiac Ultrasound: Principles, Implementation and Limitations," Eur. J. Echocardiogr., vol. 1, no. 3, pp. 154-170, Sep. 2000.

[13] G. Perk, P. A. Tunick, and I. Kronzon, "Non-Doppler Two-dimensional Strain Imaging by Echocardiography-From Technical Considerations to Clinical Applications," J. Am. Soc. Echocardiogr., vol. 20, no. 3, pp. 234243, Mar. 2007.

[14] J. J. Bax et al., "Usefulness of myocardial tissue Doppler echocardiography to evaluate left ventricular dyssynchrony before and after biventricular pacing in patients with idiopathic dilated cardiomyopathy," Am. J. Cardiol., vol. 91, no. 1, pp. 94-97, Jan. 2003.

[15] D. Notabartolo et al., "Usefulness of the peak velocity difference by tissue Doppler imaging technique as an effective predictor of response to cardiac resynchronization therapy," Am. J. Cardiol., vol. 94, no. 6, pp. 817820, Sep. 2004.

[16] C.-M. Yu et al., "Usefulness of Tissue Doppler Velocity and Strain Dyssynchrony for Predicting Left Ventricular Reverse Remodeling Response After Cardiac Resynchronization Therapy," Am. J. Cardiol., vol. 100, no. 8, pp. 1263-1270, Oct. 2007.
[17] L. Tong et al., "Wide-Angle Tissue Doppler Imaging at High Frame Rate Using Multi-Line Transmit Beamforming: An Experimental Validation In Vivo," IEEE Trans. Med. Imaging, vol. 35, no. 2, pp. 521-528, Feb. 2016.

[18] D. P. Shattuck, M. D. Weinshenker, S. W. Smith, and O. T. von Ramm, "Explososcan: A parallel processing technique for high speed ultrasound imaging with linear phased arrays," J. Acoust. Soc. Am., vol. 75, no. 4, pp. 1273-1282, 1984.

[19] O. T. von Ramm, S. W. Smith, and J. Pavy, H.G., "High-speed ultrasound volumetric imaging system. II. Parallel processing and image display," IEEE Trans. Ultrason. Ferroelectr. Freq. Control, vol. 38, no. 2, pp. 109-115, Mar. 1991.

[20] T. Hergum, T. Bjastad, and H. Torp, "Parallel beamforming using synthetic transmit beams [biomedical ultrasound imaging]," in 2004 IEEE Ultrasonics Symposium, 2004, vol. 2, pp. 1401-1404.

[21] J. Cheng and J.-Y. Lu, "Extended high-frame rate imaging method with limited-diffraction beams," IEEE Trans. Ultrason. Ferroelectr. Freq. Control, vol. 53, no. 5, pp. 880-899, May 2006.

[22] G. Montaldo, M. Tanter, J. Bercoff, N. Benech, and M. Fink, "Coherent plane-wave compounding for very high frame rate ultrasonography and transient elastography," IEEE Trans. Ultrason. Ferroelectr. Freq. Control, vol. 56, no. 3, pp. 489-506, Mar. 2009.

[23] L. Tong, H. Gao, H. F. Choi, and J. D'hooge, "Comparison of conventional parallel beamforming with plane wave and diverging wave imaging for cardiac applications: a simulation study," IEEE Trans. Ultrason. Ferroelectr. Freq. Control, vol. 59, no. 8, pp. 1654-1663, Aug. 2012.

[24] B. Denarie et al., "Coherent Plane Wave Compounding for Very High Frame Rate Ultrasonography of Rapidly Moving Targets," IEEE Trans. Med. Imaging, vol. 32, no. 7, pp. 1265-1276, Jul. 2013.

[25] B. F. Osmanski, D. Maresca, E. Messas, M. Tanter, and M. Pernot, "Transthoracic ultrafast Doppler imaging of human left ventricular hemodynamic function," IEEE Trans. Ultrason. Ferroelectr. Freq. Control, vol. 61, no. 8, pp. 1268-1275, Aug. 2014.

[26] H. Hasegawa and H. Kanai, "High-frame-rate echocardiography using diverging transmit beams and parallel receive beamforming," J. Med. Ultrason., vol. 38, no. 3, pp. 129-140, May 2011.

[27] C. Papadacci, M. Pernot, M. Couade, M. Fink, and M. Tanter, "Highcontrast ultrafast imaging of the heart," IEEE Trans. Ultrason. Ferroelectr. Freq. Control, vol. 61, no. 2, pp. 288-301, Feb. 2014.

[28] M. Correia, J. Provost, S. Chatelin, O. Villemain, M. Tanter, and M. Pernot, "Ultrafast Harmonic Coherent Compound (UHCC) Imaging for High Frame Rate Echocardiography and Shear-Wave Elastography," IEEE Trans. Ultrason. Ferroelectr. Freq. Control, vol. 63, no. 3, pp. 420-431, Mar. 2016.

[29] J. Poree, D. Posada, A. Hodzic, F. Tournoux, G. Cloutier, and D. Garcia, "High-Frame-Rate Echocardiography Using Coherent Compounding With Doppler-Based Motion-Compensation," IEEE Trans. Med. Imaging, vol. 35, no. 7, pp. 1647-1657, Jul. 2016.

[30] R. Mallart and M. Fink, "Improved imaging rate through simultaneous transmission of several ultrasound beams," in Proceedings of the SPIE, 1992, vol. 1733 , pp. $120-130$

[31] A. Drukarev, K. Konstantinides, and G. Seroussi, "Beam transformation techinques for ultrasonic medical imaging," IEEE Trans. Ultrason. Ferroelectr. Freq. Control, vol. 40, no. 6, pp. 717-726, Nov. 1993.

[32] L. Demi, A. Ramalli, G. Giannini, and M. Mischi, "In Vitro and in Vivo tissue harmonic images obtained with parallel transmit beamforming by means of orthogonal frequency division multiplexing," Ultrason. Ferroelectr. Freq. Control IEEE Trans. On, vol. 62, no. 1, pp. 230-235, Jan. 2015.

[33] L. Tong, A. Ramalli, R. Jasaityte, P. Tortoli, and J. D'hooge, "MultiTransmit Beam Forming for Fast Cardiac Imaging: Experimental Validation and In Vivo Application," IEEE Trans. Med. Imaging, vol. 33, no. 6, pp. 1205-1219, Jun. 2014

[34] A. Ramalli et al., "Multi transmit beams for fast cardiac imaging towards clinical routine," in 2016 IEEE International Ultrasonics Symposium (IUS), 2016, pp. 1-4.

[35] E. Boni et al., "ULA-OP 256: A 256-Channel Open Scanner for Development and Real-Time Implementation of New Ultrasound Methods," IEEE Trans. Ultrason. Ferroelectr. Freq. Control, vol. 63, no. 10, pp. 14881495, Oct. 2016.

[36] E. Boni et al., "Architecture of an Ultrasound System for Continuous Real-Time High Frame Rate Imaging," IEEE Trans. Ultrason. Ferroelectr. Freq. Control, vol. 64, no. 9, pp. 1276-1284, Sep. 2017.

[37] P. Santos, L. Tong, A. Ortega, L. Løvstakken, E. Samset, and J. D'hooge, "Acoustic output of multi-line transmit beamforming for fast cardiac imaging: a simulation study," IEEE Trans. Ultrason. Ferroelectr. Freq. Control, vol. 62, no. 7, pp. 1320-1330, Jul. 2015. 
[38] A. Ramalli, L. Tong, J. Luo, J. D’hooge, and P. Tortoli, "Safety of fast cardiac imaging using multiple transmit beams: Experimental verification," in Ultrasonics Symposium (IUS), 2014 IEEE International, 2014, pp. 11821185 .

[39] C. Kasai, K. Namekawa, A. Koyano, and R. Omoto, "Real-Time TwoDimensional Blood Flow Imaging Using an Autocorrelation Technique," IEEE Trans. Sonics Ultrason., vol. 32, no. 3, pp. 458-464, May 1985.

[40] B. Denarie, T. Bjastad, and H. Torp, "Multi-line transmission in 3-D with reduced crosstalk artifacts: a proof of concept study," IEEE Trans. Ultrason. Ferroelectr. Freq. Control, vol. 60, no. 8, pp. 1708-1718, Aug. 2013.

[41] S. Ricci, L. Bassi, E. Boni, A. Dallai, and P. Tortoli, "Multichannel FPGA-based arbitrary waveform generator for medical ultrasound," Electron. Lett., vol. 43, no. 24, pp. 1335-1336, 2007.

[42] Safety Group of the British Medical Ultrasound Society, "Guidelines for the safe use of diagnostic ultrasound equipment," Ultrasound, vol. 18, no. 2, pp. 52-59, Jan. 2010.

[43] G. ter Haar, Ed., The Safe use of Ultrasound in Medical Diagnosis, 3rd Edition edition. London: British Institute of Radiology, 2012.

[44] L. Tong, H. Gao, and J. D'hooge, "Multi-transmit beam forming for fast cardiac imaging-a simulation study," IEEE Trans. Ultrason. Ferroelectr. Freq. Control, vol. 60, no. 8, pp. 1719-1731, Aug. 2013.

[45] G. Matrone and A. Ramalli, "Spatial Coherence of Backscattered Signals in Multi-Line Transmit Ultrasound Imaging and Its Effect on ShortLag Filtered-Delay Multiply and Sum Beamforming," Appl. Sci., vol. 8, no. 4, p. 486, Mar. 2018.

[46] G. Zurakhov et al., "Multiline Transmit Beamforming Combined With Adaptive Apodization," IEEE Trans. Ultrason. Ferroelectr. Freq. Control, vol. 65 , no. 4, pp. 535-545, Apr. 2018.

[47] K. Ranganathan, M. K. Santy, T. N. Blalock, J. A. Hossack, and W. F. Walker, "Direct sampled I/Q beamforming for compact and very low-cost ultrasound imaging," IEEE Trans. Ultrason. Ferroelectr. Freq. Control, vol. 51, no. 9, pp. 1082-1094, Sep. 2004.

[48] P. Joos et al., "High-Frame-Rate Speckle-Tracking Echocardiography," IEEE Trans. Ultrason. Ferroelectr. Freq. Control, vol. 65, no. 5, pp. 720-728, May 2018.
[49] H. Dalen, A. Thorstensen, L. J. Vatten, S. A. Aase, and A. Stoylen, "Reference Values and Distribution of Conventional Echocardiographic Doppler Measures and Longitudinal Tissue Doppler Velocities in a Population Free From Cardiovascular DiseaseClinical Perspective," Circ. Cardiovasc. Imaging, vol. 3, no. 5, pp. 614-622, Sep. 2010.

[50] G. Matrone, A. Ramalli, A. S. Savoia, P. Tortoli, and G. Magenes, "High Frame-Rate, High Resolution Ultrasound Imaging With Multi-Line Transmission and Filtered-Delay Multiply And Sum Beamforming," IEEE Trans. Med. Imaging, vol. 36, no. 2, pp. 478-486, Feb. 2017.

[51] J. Grondin, V. Sayseng, and E. E. Konofagou, "Cardiac Strain Imaging With Coherent Compounding of Diverging Waves," IEEE Trans. Ultrason. Ferroelectr. Freq. Control, vol. 64, no. 8, pp. 1212-1222, Aug. 2017.

[52] F. Prieur, B. Dénarié, A. Austeng, and H. Torp, "Correspondence Multi-line transmission in medical imaging using the second-harmonic signal," IEEE Trans. Ultrason. Ferroelectr. Freq. Control, vol. 60, no. 12, pp. 2682-2692, Dec. 2013.

[53] J. V. Cauwenberge et al., "Assessing the Performance of Ultrafast Vector Flow Imaging in the Neonatal Heart via Multiphysics Modeling and In Vitro Experiments," IEEE Trans. Ultrason. Ferroelectr. Freq. Control, vol. 63, no. 11, pp. 1772-1785, Nov. 2016.

[54] C. Papadacci, E. A. Bunting, E. Y. Wan, P. Nauleau, and E. E. Konofagou, "3D Myocardial Elastography In Vivo," IEEE Trans. Med. Imaging, vol. 36, no. 2, pp. 618-627, Feb. 2017.

[55] S. Langeland et al., "RF-based two-dimensional cardiac strain estimation: a validation study in a tissue-mimicking phantom," IEEE Trans. Ultrason. Ferroelectr. Freq. Control, vol. 51, no. 11, pp. 1537-1546, Nov. 2004.

[56] S. Langeland et al., "Experimental validation of a new ultrasound method for the simultaneous assessment of radial and longitudinal myocardial deformation independent of insonation angle," Circulation, vol. 112, no. 14, pp. 2157-2162, Oct. 2005.

[57] P. Tortoli, M. Lenge, D. Righi, G. Ciuti, H. Liebgott, and S. Ricci, "Comparison of carotid artery blood velocity measurements by vector and standard Doppler approaches," Ultrasound Med. Biol., vol. 41, no. 5, pp. 1354-1362, May 2015. 